\title{
Evaluating the use of cell phone messaging for community Ebola syndromic surveillance in high risked settings in Southern Sierra Leone
}

\author{
Kangbai Jia ${ }^{1}$, Koroma Mohamed ${ }^{2}$
}

\begin{abstract}
1. University of Kentucky, College of Public Health, Department of Epidemiology
2. Njala University, School of Community Health Sciences, Department of Clinical and Community Health Sciences
\end{abstract}

\begin{abstract}
Background: Most underdeveloped countries do not meet core disease outbreak surveillance because of the lack of human resources, laboratory and infrastructural facilities. The use of cell phone technology for disease outbreak syndromic surveillance is a new phenomenon in Sierra Leone despite its successes in other developing countries like Sri Lanka. In this study we set to evaluate the effectiveness of using cell phone technology for Ebola hemorrhagic fever syndromic surveillance in a high risked community in Sierra Leone.

Objectives: This study evaluated the effectiveness of using cell phone messaging (text and calls) for community Ebola hemorrhagic fever syndromic surveillance in high risked community in southern Sierra Leone.

Method: All cell phone syndromic surveillance data used for this study was reported as cell phone alert messages-texts and voice calls; by the Moyamba District Health Management Team for both Ebola hemorrhagic fever suspect and mortalities. We conducted a longitudinal data analysis of the monthly cumulative confirmed Ebola hemorrhagic fever cases and mortalities collected by both the traditional sentinel and community cell phone syndromic surveillance from August 2014 to October 2014.

Results: A total of 129 and 49 Ebola hemorrhagic fever suspect and confirmed cases respectively were recorded using the community Ebola syndromic surveillance cell phone alert system by the Moyamba District Health Management Team in October 2014. The average number of Ebola hemorrhagic fever suspects and confirmed cases for October 2014 were 4.16 (Std.dev 3.76) and 1.58 (Std.dev 1.43) respectively. Thirty-four percent $(n=76)$ of the community Ebola syndromic surveillance cell phone alerts that were followed-up within 24 hours reported Ebola hemorrhagic fever suspect cases while $65.92 \%$ $(\mathrm{n}=147)$ reported mortality.

Conclusion: Our study suggests some form of underreporting by the traditional sentinel Ebola hemorrhagic fever disease surveillance system in Moyamba District southern Sierra Leone for August-September 2014. Cell phone messaging technology can be effectively use as a tool for community epidemic surveillance from peripheral health care facilities to higher levels.

Keywords: Cell phone, Community, Ebola, Syndromic, Surveillance

DOI: http://dx.doi.org/10.4314/ahs.v15i3.13

Cite as: Jia K, Mohamed K. Evaluating the use of cell phone messaging for community Ebola syndromic surveillance in high risked settings in Southern Sierra Leone. Afri Health Sci. 2015;15(3):797-802. doi: http:// dx.doi.org/10.4314/abs.v15i3.13
\end{abstract}

\section{Introduction}

Effective containment of infectious disease epidemic depends on early detection of signs and symptoms among susceptible community members. Community syndromic surveillance using cell phone messaging could reduce delays in case reporting. One key objective of the Center for Disease Control and Prevention's

\section{Corresponding author: \\ Kangbai Jia, \\ University of Kentucky, \\ College of Public Health, \\ Department of Epidemiology \\ Email: jiakangbai@hotmail.com/ \\ jia.kangbai@uky.edu}

(CDC) in 1998 was to develop a disease surveillance program for the early detection and investigation of disease outbreak ${ }^{1}$. The release of Bacillus anthracis spores through mail in 2001 led to the formulation of the United States syndromic surveillance systems ${ }^{2}$. Most syndromic surveillance systems operate on ICD10-CM codes which is based on diagnosis and medical procedures associated with hospital utilization in the United States ${ }^{3}$. ICD-10-CM codes are more useful than other health system data streams because of its data's timeliness ${ }^{4}$.

Syndromic surveillance focuses on the early symptom (prodrome) period and uses clinical and alternative data sources which it incorporates with the patient's health information relating to the early phases of an illness. Many forms of syndromic surveillance data systems 
exist. An optimal syndromic surveillance system integrates data from multiple sources thereby leading to an increased investigator's confidence ${ }^{4}$.

The first case of Ebola Hemorrhagic Fever (EHF) in Sierra Leone was documented on $27^{\text {th }}$ May 2014 in Kailahun district ${ }^{5}$. Early epidemiological studies reported it to have been imported from neighbouring Guinea ${ }^{6}$. As of December 2014, the national cumulative confirmed cases and death of EHF for Sierra Leone was 9,633 and 2,827 respectively with Moyamba District accounting for $3.01 \%$ of the national cumulative confirmed cases ${ }^{7}$.

\section{Method}

To evaluate the effectiveness of using cell phone short message service (SMS) text and voice messaging for community EHF syndromic surveillance in high risked community in southern Sierra Leone we conducted a longitudinal data analysis of monthly EHF suspect and mortality cases collected by both the traditional sentinel and community cell phone EHF syndromic surveillance for the period August 2014 to October 2014. For analysis purpose, we considered August 2014 to September 2014 as the traditional sentinel surveillance period and October 2014 as the community cell phone EHF syndromic surveillance period. Moyamba District started using community cell phone EHF syndromic surveillance in October 2014. The district was selected as our surveillance area because it recorded the highest Ebola incidence density and laboratory confirmed EHF cases and mortalities in southern Sierra Leone during the period under review.

Ethical approval for this study was obtained from the Ethical Board of Njala University in Sierra Leone. We did not collect informed consent from the study subjects because the data used in this study could not be medically linked to any personal health data bank and this data was collected as part of Moyamba District Health Management Team (MDHMT) national public health response effort to contain EVD-2014 outbreak in Sierra Leone.

\section{Moyamba District Community Syndromic Surveil- lance System}

Moyamba District has a population of $278,119^{7}$. The district has extensive uninterrupted cell phone coverage for all mobile phone companies operating in Sierra Leone. More than three million Sierra Leonean adults own cell phones while the total population that have access to cell phone is about four million people ${ }^{10}$. About $2 \%$ of Moyamba District's population has accessed to cell phone ${ }^{7}$.

The MDHMT operates an Ebola Task Force with support from the international medical charity Action Contre la Famin (ACF). The task force educate residents through various radio discussion and phone-in programs about Ebola transmission methods, control and prevention. Community members who take part in these radio programs do so in different dialects including the predominantly Mende dialect. Non-Mende contributors to these health discussion programs also have their messages translated into the common Mende dialect for better understanding and later into English for record keeping purpose.

Moyamba District community members with access to cell phone can also communicate through SMS messaging to the Ebola syndromic surveillance officers and volunteers or directly to the MDHMT Ebola Task Force headquarters issues relating to Ebola outbreak in their various community. Community members who cannot write English can have a literate to report on the existence of Ebola suspects or mortality in their community through SMS messaging.

For easy and effective EHF surveillance Moyamba District was divided into three zones with each zone headed by an Ebola syndromic surveillance zonal commander. For this study, community Ebola surveillance includes reporting of EHF suspect or mortality by community members, Ebola syndromic surveillance officers, and community Ebola surveillance volunteers to the Moyamba District EHF surveillance headquarters. Upon receiving a report about an EHF suspect or death, disease outbreak investigators from the MDHMT disease surveillance headquarters will evacuate the Ebola suspect to an Ebola treatment center. If the case is deceased, an Ebola burial team will be dispatched to pick up the body for safe burial.

This study considered an EHF case as an Ebola suspect which was confirmed positive through various laboratory diagnosis including but not restricted to PCR and viral isolation. An EHF suspect is defined as someone who is ill with onset of fever and not responding to treatment for usual causes of fever in the area, and has at least one of the following signs: bloody diarrhoea, bleeding from gums, bleeding into skin (purpura), bleeding into eyes and urine ${ }^{8}$. 


\section{Study site and data flow}

Sierra Leone is divided into 13 districts with the Western Area Rural and Western Area Urban district collectively forming the Western Area Health District. There is a DHMT in each district containing at least 50 peripheral health units (PHU) and over 100 technical staff that are responsible for planning, organizing and monitoring health provision, training healthcare personnel, working with communities and supplying equipment and drugs 9 A National Ebola Response Center (NERC) formed by the Ministry of Health and Sanitation (MOHS) coordinates, monitors and evaluates the various national EHF containment measures. The NERC's Ebola surveillance system data originates from the DHMT level and flows through the provincial level to the national level in the capital Freetown. It is based on a weekly MS Excel information bulletin system which starts from the Maternal Child Health Post and goes through the Community Health Post to the Community Health Center and finally to the DHMT.

\section{Data quality assurance}

Data analysis for this study underwent three quality assessments: primary contact level assessment, secondary assessment at DHMT briefing, and a follow up assessment action. For effective contact tracing EHF syndromic surveillance officers record the following variables; age, sex, name and location of suspect or deceased case with each variable checked at each assessment level. To prevent recall bias community cell phone EHF syndromic surveillance alerts were also recorded on MS Excel sheet and later traced on a white board. All community cell phone EHF syndromic surveillance alerts for both Ebola suspect or mortality case were followed-up for confirmation by a Community Health Officer and an EHF contact tracer after they had been recorded at the Moyamba District EHF syndromic surveillance headquarters.

\section{Analysis}

We determined the descriptive statistics for both Ebola surveillance periods and compared the optimum EHF surveillance data collected for all types of EHF cases and mortalities for the review periods. Cell phone syndromic surveillance data for this study were reported as cell phone SMS text or voice messages by the MDHMT for both EHF suspect and mortality cases. Health cases and population indicators not related to EHF were not included in this analysis. Student's t-test was used to determine the statistical significance of the result for this study.

\section{Results}

A total of 129 EHF suspect cases and 199 EHF mortalities were recorded by the Moyamba DHMT in October 2014 using the community syndromic surveillance cell phone alerts system. The average number of EHF suspects and confirmed cases for October 2014 were 4.16 (Std.dev 3.76) and 1.58 (Std.dev 1.43) respectively. The number of EHF suspect cases reported using cell phone syndromic surveillance alerts messaging for October 2014 were statistically significant at $0.05 \mathrm{lev}$ el (t-test 3.499, p-value 0.000443). The average number EHF mortalities reported by cell phone syndromic surveillance system was 6.42 (Std.dev 3.91, 95\% CI 5.88-6.94). The number of EHF mortalities reported using cell phone syndromic surveillance alerts messaging for October 2014 were also statistically significant at 0.05 level (t-test 4.323, p-value 0.003443). There was a strong positive linear correlation ( $\mathrm{R}$ 0.8493) between cell phone syndromic surveillance reported EFH suspect cases and deaths when data was stratified for chiefdoms in Moyamba District (Table 1). There was also a strong positive linear correlation ( $\mathrm{R} 0.8055)$ between cell phone syndromic surveillance data for EFH suspect cases and seropositivity in October 2014. 
Table 1. Adjusted cellphone syndromic data alerts for EHF suspects, deaths and laboratory findings by chiefdom in Moyamba District for October 2014

\begin{tabular}{|c|c|c|c|c|c|c|c|c|c|}
\hline Chiefdom & \begin{tabular}{|l} 
Suspect \\
EHF \\
cases
\end{tabular} & $\begin{array}{l}\text { Suspect } \\
\text { EHF } \\
\text { deaths }\end{array}$ & Total & \begin{tabular}{l|} 
Lab \\
positive \\
suspected \\
case
\end{tabular} & $\begin{array}{l}\text { Positive } \\
\% \\
\text { among } \\
\text { suspects }\end{array}$ & \begin{tabular}{|l} 
Lab \\
positive \\
dead \\
case \\
\end{tabular} & $\begin{array}{l}\text { Positive } \\
\% \\
\text { among } \\
\text { death } \\
\end{array}$ & $\begin{array}{l}\text { Positive } \\
\text { total }\end{array}$ & $\begin{array}{l}\text { Positive } \\
\%\end{array}$ \\
\hline Bagruwa & 2 & 10 & 12 & 0 & 0 & 0 & 0 & 0 & 0 \\
\hline Bumpeh & 5 & 18 & 23 & 0 & 0 & 0 & 0 & 0 & 0 \\
\hline Dasse & 0 & 13 & 13 & 0 & 0 & 0 & 0 & 0 & 0 \\
\hline Fakunya & 26 & 23 & 49 & 8 & 30.8 & 0 & 0 & 8 & 16.0 \\
\hline Kaiyamba & 25 & 26 & 51 & 3 & 12.0 & 0 & 0 & 3 & 5.9 \\
\hline Kamajei & 2 & 2 & 4 & 0 & 0 & 0 & 0 & 0 & 0 \\
\hline Kargboro & 5 & 18 & 23 & 2 & 40.0 & 0 & 0 & 2 & 8.7 \\
\hline Kombora & 0 & 7 & 7 & 0 & 0 & 0 & 0 & 0 & 0 \\
\hline Kori & 14 & 19 & 33 & 7 & 50.0 & 3 & 15.8 & 10 & 30.3 \\
\hline Kowa & 0 & 4 & 4 & 0 & 0 & 0 & 0 & 0 & 0 \\
\hline $\begin{array}{l}\text { Lower } \\
\text { Banta }\end{array}$ & 30 & 23 & 53 & 18 & 60.0 & 0 & 0 & 18 & 34.0 \\
\hline Ribbi & 20 & 26 & 46 & 11 & 55.0 & 3 & 11.5 & 14 & 29.8 \\
\hline Timadale & 0 & 8 & 8 & 0 & 0 & 0 & 0 & 0 & 0 \\
\hline $\begin{array}{l}\text { Upper } \\
\text { Banta }\end{array}$ & 0 & 2 & 2 & 0 & 0 & 0 & 0 & 0 & 0 \\
\hline TOTAL & 129 & 199 & 328 & 49 & 38.0 & 6 & 3.0 & 55 & 16.5 \\
\hline
\end{tabular}

Out of the 260 community syndromic surveillance cell phone alerts made in October 2014, 85.77\% $(\mathrm{n}=223)$ were successfully followed up within a day $(<24 \mathrm{hrs})$, $13.85 \%(\mathrm{n}=36)$ were unmet calls, while $0.38 \%(\mathrm{n}=1)$ was false alert (Table 2). Thirty-four percent $(n=76)$ of the alerts that were followed-up within $24 \mathrm{hrs}$ report- ed EHF suspect cases while 65.92\% ( $n=147)$ reported mortality. The number of suspect EHF cases reported from these alerts were slightly positively correlated $(\mathrm{R}$ 0.0537) with EHF mortality. The percentage of $<24 \mathrm{hrs}$ cell phone syndromic surveillance alerts received for EHF mortality $(65.9 \%)$ was almost twice that for suspects $(34.1 \%)$ cases.

Table 2. Cellphone alerts distribution by EHF suspects and death October 2014

\begin{tabular}{|c|c|c|c|c|c|c|}
\hline \multirow{3}{*}{$\begin{array}{l}\begin{array}{l}\text { Type of } \\
\text { Alert }\end{array} \\
<24 \mathrm{hr}\end{array}$} & \multicolumn{2}{|c|}{ No. of Alert } & \multirow{3}{*}{\begin{tabular}{|l} 
Suspect \\
76 \\
\end{tabular}} & \multirow{3}{*}{$\begin{array}{l}\% \\
34.08\end{array}$} & \multicolumn{2}{|c|}{ Death } \\
\hline & \multirow[b]{2}{*}{223} & \multirow{2}{*}{\begin{tabular}{|l|}
$\%$ \\
85.77 \\
\end{tabular}} & & & \multirow[b]{2}{*}{147} & \multirow{2}{*}{\begin{tabular}{|l|}
$\%$ \\
65.92
\end{tabular}} \\
\hline & & & & & & \\
\hline $\begin{array}{l}\text { Unmet } \\
\text { alert }\end{array}$ & 36 & 13.85 & 14 & 38.89 & 5 & 13.89 \\
\hline $\begin{array}{l}\text { False } \\
\text { Alert }\end{array}$ & 1 & 0.38 & 1 & -- & 0 & -- \\
\hline Total & 260 & 100 & 103 & -- & 157 & -- \\
\hline
\end{tabular}


Fifty EHF confirmed cases and 4 EHF mortalities were recorded by the Moyamba DHMT using the traditional sentinel disease surveillance for August-September 2014 (Table 3). The average number of EHF suspect and confirmed cases reported by the traditional sentinel disease surveillance for August-September 2014 were 1.14 (Std.dev 2.47) and 1.07(Std.dev 2.31) respectively. The Student t-test for daily EHF suspect cases for August-September 2014 was non significance at 0.05 level (t-test1.146, p-value 0.13).

Table 3. Monthly EHF cases and mortality for Moyamba District August-November 2014

\begin{tabular}{|l|l|l|l|}
\hline Months & $\begin{array}{l}\text { EHF confirmed } \\
\text { cases }\end{array}$ & EHF mortality & Total \\
\hline August & & 1 & 5 \\
\hline September & 4 & 1 & 49 \\
\hline October & 46 & 3 & 63 \\
\hline
\end{tabular}

There was weak correlation between the traditional sentinel surveillance data for suspect cases and deaths $(r=0.0013)$ when data was stratified for chiefdoms in Moyamba District for August-September 2014 and correlation was also weak $(r=0.00024)$ for $\mathrm{EFH}$ suspect cases and seropositivity.

According to the NERC, the cumulative confirmed cases and mortality for EHF Moyamba District for the months of August, September, October, November and 2014 were: 4 (death=1), $46($ death=3), $38($ death=25), $64($ death $=8)$, and 25 (death=3) respectively ${ }^{7}$.

\section{Discussion}

The use of cell phone technology to monitor epidemic outbreak though a novelty in Sierra Leone yet seems to be a promising syndromic surveillance tool. More than one million Sierra Leonean adults own a cell phone ${ }^{10}$. Community syndromic surveillance by cell phone has major advantages including its scalability which enable large number of susceptible people to be monitored at the same time thereby reducing the cost of additional surveillance.

Out of the 260 community syndromic surveillance cell phone alerts made in October 2014, there were $55($ EHF positive cases=49, EHF positive deaths=6) laboratory positive EHF results which is slightly high- er than the combined number of positive EHF cases for August and September 2014. The total laboratory positive EHF results recorded for August 2014 and September 2014 was 54 (EHF positive cases=50, EHF positive deaths $=4)^{7}$. Although this study only analysed EHF cases and mortality recorded by the community cell phone syndromic surveillance system during the review period, our study tends to suggest some form of under reporting by the traditional sentinel EHF disease surveillance system.

The surprisingly effective and timely reporting of EHF suspect cases and deaths demonstrated by the increased number of confirmed EHF cases and incidence in October 2014 in our study compared to the period prior to the use of community cell phone syndromic surveillance system forces the need for further exploration about the accuracy and widespread use of cell phone technology in monitoring infectious disease outbreak in resource-poor countries.

Our study did not factor the significance of unmet (alerts that were not followed up) alerts which accounted for $13.85 \%$ of the monthly syndromic surveillance cell phone alerts on the number of EHF suspect cases and incidence identified in November 2014. Future studies should look into importance of unmet and false alerts on the incidence and confirmed suspect cases of EHF as well as the impact of stratifying community 
syndromic surveillance cell phone alerts data into sex, age and locations.

\section{Conclusion}

Our study shows that the use of cell phone messaging as a tool for community epidemic surveillance from peripheral health care facilities to higher levels is possible. Cell phone technology can provide important clues and data for public health policy and practice. In the broader sense resource-poor countries will use it to develop real-time indices for epidemic control in order to understand the patterns and trends that are important for their population health. National public health systems can also upgrade the ways into which cell phone syndromic surveillance can be put into use to also serve as early warning system for health issues such as the outbreak of epidemics and emerging diseases, as well as that of bioterrorism.

Some major advantages of using cell phone community syndromic surveillance system to monitor EHF epidemic is its scalability; that is it enables large number of susceptible people to be monitored at the same time and thereby reduced the cost of additional surveillance. However in spite of these potential positive public health outcomes for the use of cell phone messaging in epidemic situation, the accuracy of its data should be validating in the future. Future studies should target on improving the quality of cell phone data reported during syndromic surveillance.

\section{Consent}

Not applicable.

\section{Ethical approval}

This study was approved by the Ethical Board of Njala University in Sierra Leone.

\section{Acknowledgement}

We will like to specially acknowledge the contribution of Mohamed Kallon the Deputy Monitoring Officer of Action Contre le Faim in Sierra Leone for allowing us to use community syndromic surveillance data for Ebola which we used in this work.

\section{Competing interests}

None.

\section{Reference}

1. CDC (2004). Preventing emerging infectious diseases: a strategy for the 21st century. Atlanta, GA: US Department of Health and Human Services. Accessed January 2015. Available at http://www.cdc.gov/ncidod/emergplan/index.htm.

2. CDC. Update: investigation of anthrax associated with intentional exposure and interim public health guidelines, October 2001. MMWR 2001;50:889--93 PubMed .

3. Daniel Dajun Zeng et al, (2014); Syndromic surveillance systems.

4. Henning K.J (2013); Overview of Syndromic Surveillance What is Syndromic Surveillance?

5. Ebola Virus Disease Masanga Hospital Rehabilitation Project Tonkolili District, Sierra Leone MarchAugust 2014. Accessed January 2015 Available http:// globalhealth.ku.dk/events/2014/ebola_experiences_ lessons_learnt/EVD_Masanga2014.pdf

6. ECDC (2014). Epidemiological updates :outbreak of Ebola virus disease in West Africa. Accessed January 2015. Available http://www.ecdc.europa.eu/en/ healthtopics/ebola_marburg_fevers/Pages/ebola-outbreak-west-africa.aspx

7. Ebola Virus Disease- Situation Report (Sit-Rep) 01 JANUARY, 2015. Accessed January 2015. Available http://health.gov.sl/wp-content/uploads/2015/01/ Ebola-Situation-Report_Vol-218.pdf

8. WHO. Case definition recommendations for Ebola or Margburg Virus Disease (2014). Accessed January 2015. Available http://www.who.int/csr/resources/ publications/ebola/ebola-case-definition-contact-en. pdf

9. Ministry of Health and Sanitation. National Health Sector Strategic Plan 2010- 2015 Joint Program of Work and Funding (JPWF) 2012 -2014 PubMed Accessed January 2015. Available http://www.whosierraleone.org/1_docs/mohspartnersdocs/jpwf_final.pdf 10. Global Resources and Information Directory. Accessed January 2015. Available http://www.fosigrid. org/africa/sierra-leone. 